\title{
THE REGULARITY OF WEAK AND VERY WEAK SOLUTIONS OF THE POISSON EQUATION ON POLYGONAL DOMAINS WITH MIXED BOUNDARY CONDITIONS (PART I)
}

Abstract. We examine the regularity of weak and very weak solutions of the Poisson equation on polygonal domains with data in $L^{2}$. We consider mixed Dirichlet, Neumann and Robin boundary conditions. We also describe the singular part of weak and very weak solutions.

1. Introduction. In this series of two papers we describe the regularity of weak and very weak solutions of the Poisson equation on polygonal domains. We consider mixed boundary conditions of the following types: Dirichlet, Neumann and Robin. We also characterize the singular part of weak and very weak solutions. By a very weak solution we mean a function from the maximal domain of the Laplace operator in $L^{2}$ which satisfies the Poisson equation. In our investigations of the regularity of solutions we follow Grisvard [5]. Our theorems generalize some results of [4] and [5]. Our main contribution is in the case of Robin boundary conditions with constant coefficients.

Let us recall that if we consider the Poisson equation on a smooth domain with data from $L^{2}$, then the solution is in $H^{2}$. However, if the domain is not smooth or if we admit mixed boundary conditions, then we may obtain singular solutions, i.e. not in $H^{2}$. In this case, it is important to characterize the asymptotic behaviour of the solution in a neighbourhood of a singular point. A powerful tool to study this is Kondrat'ev's theory [6]. It allows us to describe the asymptotic behaviour of solutions of the Poisson equation with data from a weight space, where the weight is a power of the distance from the conical point. The regularity of weak solutions of mixed Dirichlet

2000 Mathematics Subject Classification: 35B40, 35B65, 35C20, 35J05, 35J25.

Key words and phrases: Poisson equation, polygonal domain, mixed boundary value problem, regularity of weak and very weak solutions. 
(D), Neumann (N) and Robin (R) boundary value problems may be deduced from [7, Theorem 6.4.1], for example, provided that the angles are different from the exceptional values. However, Grisvard's approach (see [4], [5]) covers those exceptional cases and also allows us to characterize the singular behaviour of very weak solutions. This cannot be obtained from Kondrat'ev's theory. We want to stress that the regularity of weak solutions of mixed $\mathbf{D}, \mathbf{N}, \mathbf{R}$ boundary value problems may be deduced from [5, Theorems 1.4.6 and 2.4.3], but we have not been able to obtain the analogous result for very weak solutions using [5]. In order to attain it we have to adjust Grisvard's approach to the Robin condition.

There is a vast body of literature devoted to the smoothness of solutions of elliptic boundary value problems on nonsmooth domains. Let us only mention some monographs: [4], [13], [5], [11], [2], [7]. However, most of these monographs deal with $\mathbf{D}, \mathbf{N}$ or mixed $\mathbf{D}-\mathbf{N}$ boundary conditions. Mixed D-R, N-R boundary conditions are studied to a lesser extent. They may be found e.g. in [10] and [1]. However, in the latter article the author assumes that the coefficient in the Robin condition vanishes at a vertex. This assumption excludes the Robin condition with a constant coefficient. On the other hand, very weak solutions were studied in [3], but their definition in that paper is different from ours. Our notion of very weak solution is based on the terminology of [4]. The results presented in this paper come from [8] and the details which are omitted here may be found in [8].

This series of two papers is organized as follows. In the present one we investigate the properties of the Laplace operator restricted to the subspace of $H^{2}(\Omega)$ defined by homogeneous boundary conditions. We prove that this operator is injective and has a closed range. Then we characterize the annihilator of its range as a subspace of the space of very weak solutions of the homogeneous problem, defined by some orthogonality conditions. At the beginning of the second paper we examine the very weak solutions of the homogeneous problem. We prove some results on their smoothness and then we obtain their series expansion of a special form. This allows us to calculate the dimension of the space of very weak solutions of the homogeneous problem. Next, we prove a result on the regularity of weak and very weak solutions of the Poisson equation with homogenous boundary conditions. In the last part of the second paper we formulate analogous results for nonhomogeneous boundary conditions.

2. Assumptions and the main results. We consider a polygonal domain $\Omega$ in $\mathbb{R}^{2}$. Its boundary is composed of segments $\bar{\Gamma}_{j}, j=1, \ldots, N$, where $\Gamma_{j}$ is an open segment with endpoints $S_{j}$ and $S_{j+1}$. We assume that $\bar{\Gamma}_{k} \cap \bar{\Gamma}_{j}=\emptyset$ for $k \neq j-1, j, j+1$ and $\bar{\Gamma}_{j-1} \cap \bar{\Gamma}_{j}=\left\{S_{j}\right\}$ for $j=1, \ldots, N$. We denote by $\omega_{j}$ the angle between $\Gamma_{j-1}$ and $\Gamma_{j}$ and we assume that $\omega_{j} \in(0,2 \pi)$. 
The segments are numbered in such a way that $\Gamma_{j-1}$ follows $\Gamma_{j}$ in the positive orientation of the plane. We denote by $\tau_{j}$ the unit tangent vector to $\Gamma_{j}$ and by $\nu_{j}$ the unit outward normal vector to $\Gamma_{j}$ such that the orientation of the pair $\left(\tau_{j}, \nu_{j}\right)$ is positive. Furthermore, we attach to each vertex $S_{j}, j=$ $1, \ldots, N$, the polar (resp. cartesian) coordinates $\left(r_{j}, \theta_{j}\right)$ (resp. $\left.\left(x_{j}, y_{j}\right)\right)$ such that $\Gamma_{j-1} \subseteq\left\{\left(r_{j}, \theta_{j}\right) ; r_{j}>0, \theta_{j}=0\right\}$ and $\Gamma_{j} \subseteq\left\{\left(r_{j}, \theta_{j}\right) ; r_{j}>0, \theta_{j}=\omega_{j}\right\}$ (resp. $\Gamma_{j-1} \subseteq\left\{\left(x_{j}, y_{j}\right) ; x_{j}>0, y_{j}=0\right\}$ and $\Gamma_{j} \subseteq\left\{\left(x_{j}, y_{j}\right) ; x_{j} \sin \omega_{j}=\right.$ $\left.\left.y_{j} \cos \omega_{j}\right\}\right)$.

For $|\sigma| \leq \min _{j=1, \ldots, N}\left|\Gamma_{j}\right|$ we denote by $x_{j}(-\sigma)$ (resp. $x_{j}(\sigma)$ ) the point of $\bar{\Gamma}_{j-1}\left(\right.$ resp. $\left.\bar{\Gamma}_{j}\right)$ which is at distance $\sigma$ from $S_{j}$. We fix a partition of $\{1, \ldots, N\}$ into three subsets $\mathbf{D}, \mathbf{N}, \mathbf{R}$. We consider the following mixed boundary value problem:

$$
\begin{cases}\Delta u=f & \text { in } \Omega \text { for } f \in L^{2}(\Omega), \\ \gamma_{j} u=0 & \text { on } \Gamma_{j} \text { for } j \in \mathbf{D}, \\ \gamma_{j} \frac{\partial u}{\partial \nu_{j}}=0 & \text { on } \Gamma_{j} \text { for } j \in \mathbf{N}, \\ \gamma_{j} \frac{\partial u}{\partial \nu_{j}}+\alpha_{j} \gamma_{j} u=0 & \text { on } \Gamma_{j} \text { for } j \in \mathbf{R},\end{cases}
$$

where $\gamma_{j}$ is the trace operator on $\Gamma_{j}$. We assume that $\alpha_{j}>0$ for $j \in \mathbf{R}$ and we are not dealing with the pure Neumann problem, i.e. $\mathbf{D} \cup \mathbf{R} \neq \emptyset$, because the regularity of solutions of the Neumann problem is well known (see [5]). The former assumption guarantees the uniqueness of variational solutions of problem (2.1). Furthermore, we assume that if $j-1, j \in \mathbf{N} \cup \mathbf{R}$, then $\omega_{j} \neq \pi$.

We recall the standard definitions of the Sobolev spaces. We follow Grisvard [4]. For $m \in \mathbb{N}, p \geq 1$ and $U$ an open subset of $\mathbb{R}^{n}$ with Lipschitz boundary we denote by $W^{m, p}(U)$ the space of distributions $u$ on $U$ such that $D^{\alpha} u(x) \in L^{p}(U)$ for $|\alpha| \leq m$ with the norm $\|u\|_{W^{m, p}(U)}:=$ $\left\{\sum_{|\alpha| \leq m} \int_{U}\left|D^{\alpha} u(x)\right|^{p} d x\right\}^{1 / p}$. For each $s \in \mathbb{R}_{+} \backslash \mathbb{N}$ there exist $m \in \mathbb{N}$ and $\sigma \in(0,1)$ such that $s=m+\sigma$ and then the space $W^{s, p}(U)$ is defined to consist of those functions from $W^{m, p}(U)$ which satisfy

$$
\iint_{U} \frac{\left|D^{\alpha} u(x)-D^{\alpha} u(y)\right|^{p}}{|x-y|^{n+\sigma p}} d x d y<\infty \quad \text { for }|\alpha|=m .
$$

The norm in $W^{s, p}(U)$ is defined by

$$
\|u\|_{W^{s, p}(U)}=\left\{\|u\|_{W^{m, p}(U)}^{p}+\sum_{|\alpha|=m} \iint_{U} \frac{\left|D^{\alpha} u(x)-D^{\alpha} u(y)\right|^{p}}{|x-y|^{n+\sigma p}} d x d y\right\}^{1 / p} .
$$

In the case $p=2$, the space $W^{s, 2}(U)$ is also denoted by $H^{s}(U)$. In addition, we need another Sobolev space, $\widetilde{H}^{s}(U):=\left\{u \in H^{s}(U) ; \widetilde{u} \in H^{s}\left(\mathbb{R}^{n}\right)\right\}$, 
where $\widetilde{u}$ is the extension of $u$ by zero outside $U$. In $\widetilde{H}^{s}(U)$ we have the norm $\|u\|_{\widetilde{H}^{s}(U)}=\|\widetilde{u}\|_{H^{s}\left(\mathbb{R}^{n}\right)}$. We investigate the maximal domain of the Laplace operator in $L^{2}(U)$, i.e. $D\left(\Delta, L^{2}(U)\right):=\left\{u \in L^{2}(U) ; \Delta u \in L^{2}(U)\right\}$, equipped with the graph norm $\|u\|_{D\left(\Delta, L^{2}(U)\right)}=\left\{\|u\|_{L^{2}(U)}^{2}+\|\Delta u\|_{L^{2}(U)}^{2}\right\}^{1 / 2}$. By $E\left(\Delta, L^{2}(U)\right)$ we denote the subspace of $H^{1}(U)$, consisting of the functions such that $\Delta u \in L^{2}(U)$. This space is also equipped with the graph norm $\|u\|_{E\left(\Delta, L^{2}(U)\right)}=\left\{\|u\|_{H^{1}(U)}^{2}+\|\Delta u\|_{L^{2}(U)}^{2}\right\}^{1 / 2}$.

We use the standard symbol $\mathcal{D}(U)$ for the space of smooth functions with compact support in $U$. We write $\mathcal{D}(\bar{U})$ for the set of restrictions to $U$ of smooth functions with compact support on $\mathbb{R}^{n}$. The closures of $\mathcal{D}(U)$ in the norms of $W^{s, p}(U)$ and $H^{s}(U)$ will be denoted by $W_{0}^{s, p}(U)$ and $H_{0}^{s}(U)$ respectively. For $p \geq 1$ and $\alpha \in \mathbb{R}$ we denote by $L_{p, \alpha}\left(\mathbb{R}_{+}\right)$the space of measurable functions on $\mathbb{R}_{+}$such that $\int_{0}^{\infty}\left|u(t) t^{\alpha}\right|^{p} d t$ is finite.

We recall some standard notation of functional analysis. For a Hilbert space $H$ we will denote by $H^{*}$ its dual, i.e. the space of linear, continuous functionals on $H$. If $f \in H^{*}$, then its evaluation at $g \in H$ will be denoted by $\langle g, f\rangle$. In the case of Sobolev spaces, the dual of $H_{0}^{s}(U)$ will also be denoted by $H^{-s}(U)$. For $m \geq 2$ we define

$T^{m}(\Omega):=\left\{u \in H^{m}(\Omega) ; u\right.$ satisfies the boundary conditions of $\left.(2.1)\right\}$.

Finally, we set

$$
\mathcal{M}:=\left\{v \in D\left(\Delta, L^{2}(\Omega)\right) ; \Delta v=0\right. \text { and }
$$

$v$ satisfies the boundary conditions of $(2.1)\}$.

The traces of elements of $\mathcal{M}$ are well defined because for $v \in D\left(\Delta, L^{2}(\Omega)\right)$ we have $\gamma_{j} v \in \widetilde{H}^{1 / 2}\left(\Gamma_{j}\right)^{*}$ and $\gamma_{j} \frac{\partial}{\partial \nu_{j}} v \in \widetilde{H}^{3 / 2}\left(\Gamma_{j}\right)^{*}$ (see [5, Theorem 1.5.2]).

Now we are in a position to state the main results of the present paper. Let us formulate a theorem on the regularity of weak (i.e. variational) solutions of the mixed boundary value problem (2.1).

THEOREM 1. There exists a constant $C$, an integer $K$ and family of functions $\left\{S_{k}\right\}_{k=1}^{K} \subseteq H^{1}(\Omega) \backslash H^{2}(\Omega)$ with the following property. For every $f \in L^{2}(\Omega)$ there exists a unique $u \in H^{1}(\Omega)$ and numbers $a_{k} \in \mathbb{R}, k=$ $1, \ldots, K$, such that $u$ is a variational solution of problem (2.1) and

$$
\begin{gathered}
u-\sum_{k=1}^{K} a_{k} S_{k} \in H^{2}(\Omega), \\
\left\|u-\sum_{k=1}^{K} a_{k} S_{k}\right\|_{H^{2}(\Omega)}+\sum_{k=1}^{K}\left|a_{k}\right| \leq C\|f\|_{L^{2}(\Omega)} .
\end{gathered}
$$

Concerning very weak solutions we will prove the following theorem. 
THEOREM 2. There exists an integer $\bar{K}$ and a family of harmonic functions $\left\{F_{k}\right\}_{k=1}^{\bar{K}} \subseteq D\left(\Delta, L^{2}(\Omega)\right) \backslash H^{1}(\Omega)$ with the following property. For each solution $v \in D\left(\Delta, L^{2}(\Omega)\right)$ of problem (2.1) there exist unique numbers $c_{k} \in \mathbb{R}, k=1, \ldots, \bar{K}$, and a function $u \in H^{1}(\Omega)$ such that $u$ satisfies (2.1) and

$$
v=u+\sum_{k=1}^{\bar{K}} c_{k} F_{k} .
$$

REMARK 1 . The numbers $K, \bar{K}$ and the asymptotic behaviour of the functions $S_{k}, F_{k}$ will be described in Remarks 5 and 6 of [9].

Since the proof of Theorem 1 is the principal part of this series of papers, we shall briefly comment on its structure. The proof will be given in four steps. First, we prove that the Laplace operator transforms $T^{2}(\Omega)$ onto a closed subspace of $L^{2}(\Omega)$. Subsequently, we describe $\mathcal{N}:=\Delta T^{2}(\Omega)^{\perp}$ as a subspace of $\mathcal{M}$, defined by some orthogonality conditions. These results are established in this paper. In [9] we will calculate $\operatorname{dim} \mathcal{N}=: K$ and $\operatorname{dim} \mathcal{M}=: \bar{K}$. Finally, we will define the linearly independent family $\left\{S_{k}\right\}_{k=1}^{K}$ $\subseteq H^{1}(\Omega) \backslash H^{2}(\Omega)$ such that $\mathcal{N}=\operatorname{span}\left\{\Delta S_{k} ; k=1, \ldots, K\right\}$. These results lead to Theorem 1 . Theorem 2 will be deduced from the proof of the former.

3. The semi-Fredholm property. The main purpose of this section is to show that the Laplace operator transforms $T^{2}(\Omega)$, which is a subspace of $H^{2}(\Omega)$, onto a closed subset of $L^{2}(\Omega)$. We also prove that there exists a unique variational solution of problem (2.1); this guarantees that the Laplace operator restricted to $T^{2}(\Omega)$ is injective. Thus, we shall prove that the operator $\Delta: T^{2}(\Omega) \rightarrow L^{2}(\Omega)$ has the semi-Fredholm property. Furthermore, we shall show that the subspace of smooth functions in $T^{2}(\Omega)$ is dense in $T^{2}(\Omega)$. This will be useful later.

Now we demonstrate the following theorem.

Theorem 3. The operator $\Delta: T^{2}(\Omega) \rightarrow L^{2}(\Omega)$ is injective and has a closed range.

Proof. We shall show that there exists a unique variational solution of problem (2.1). First, we introduce some notation. For $\varepsilon>0$ we set $\Omega_{\varepsilon}:=$ $\Omega \backslash \bigcup_{j=1}^{N} B\left(S_{j}, \varepsilon\right)$, where $B\left(S_{j}, \varepsilon\right)$ stands for the ball with center $S_{j}$ and radius $\varepsilon$. Furthermore, we define $\mathcal{D}_{S}:=V_{s} \cap \mathcal{D}(\bar{\Omega})$, where

$$
\begin{aligned}
V & :=\left\{u \in H^{1}(\Omega) ; \gamma_{j} u=0 \text { for } j \in D\right\}, \\
V_{s} & :=\left\{u \in V ; \operatorname{supp} u \subseteq \Omega_{\varepsilon} \text { for some } \varepsilon>0\right\} .
\end{aligned}
$$


We define a bilinear form on $V$ by

$$
(u, v)_{V}:=\int_{\Omega} \nabla u \cdot \nabla v d x d y+\sum_{j \in \mathbf{R}} \alpha_{j} \int_{\Gamma_{j}} \gamma_{j} u \gamma_{j} v d \sigma .
$$

Applying [12, Theorem 1.9] we can show that $\|\cdot\|_{V}:=\sqrt{(\cdot, \cdot)_{V}}$ is a norm on $V$ and $\left(V,(\cdot, \cdot)_{V}\right)$ is a Hilbert space. From the Riesz theorem we have:

Proposition 1. For each $f \in V^{*}$ there exists a unique $u_{f} \in V$ such that

$$
\left(u_{f}, w\right)_{V}=\langle w, f\rangle \quad \text { for } w \in V .
$$

Using the density of $V_{s}$ in $V([5$, Lemma 2.1.2]) and the Green formula ([5, Theorem 1.5.4]) we can show that if $f \in L^{2}(\Omega)$, then $u_{f}$ is a solution of (2.1). The boundary conditions are well defined, because $u_{f} \in E\left(\Delta, L^{2}(\Omega)\right)$ (see [5, Theorem 1.5.4]). Hence Proposition 1 implies that $\Delta: T^{2}(\Omega) \rightarrow$ $L^{2}(\Omega)$ is injective.

In order to prove that $\Delta T^{2}(\Omega)$ is a closed subspace of $L^{2}(\Omega)$, we apply Peetre's lemma (see [4, Lemma 4.4.1.1]) to the Banach spaces $T^{2}(\Omega)$ and $L^{2}(\Omega)$ and the Laplace operator. Thus we have to show that there exists a constant $C$ such that

$$
\|u\|_{H^{2}(\Omega)} \leq C\left\{\|\Delta u\|_{L^{2}(\Omega)}+\|u\|_{L^{2}(\Omega)}\right\} \quad \text { for } u \in T^{2}(\Omega) .
$$

The proof of (3.4) consists of two steps. First, we show that (3.4) holds for smooth functions. Next, we prove that the smooth functions are dense in $T^{2}(\Omega)$. More precisely, we establish the following lemmas.

LEMMA 1. There exists a constant $C$ such that for all $m \geq 3$ and for all $u \in T^{m}(\Omega)$ we have the estimate

$$
\|u\|_{H^{2}(\Omega)} \leq C\left\{\|\Delta u\|_{L^{2}(\Omega)}+\|u\|_{H^{1}(\Omega)}\right\} .
$$

LemMa 2. For every $m \geq 3$ the subset $T^{m}(\Omega)$ is dense in $T^{2}(\Omega)$, i.e. $\overline{T^{m}(\Omega)} H^{2}(\Omega)=T^{2}(\Omega)$.

Applying interpolation inequalities to (3.5), and then using Lemma 2, we obtain (3.4). Hence, the proof of Theorem 3 will be finished if we prove Lemmas 1 and 2. This will be done in Subsections 3.1 and 3.2, respectively.

3.1. Proof of Lemma 1. We only have to show that there exists a constant $C$ such that for $m \geq 3$ we have

$$
\sum_{|\alpha|=2}\left\|D^{\alpha} u\right\|_{L^{2}(\Omega)}^{2} \leq C\left\{\|\Delta u\|_{L^{2}(\Omega)}^{2}+\|u\|_{H^{1}(\Omega)}^{2}\right\}
$$

for all $u \in T^{m}(\Omega)$. Integrating by parts we obtain 


$$
\begin{aligned}
&\left\|D_{x}^{2} u\right\|_{L^{2}(\Omega)}^{2}+2\left\|D_{x} D_{y} u\right\|_{L^{2}(\Omega)}^{2}+\left\|D_{y}^{2} u\right\|_{L^{2}(\Omega)}^{2} \\
&=\|\Delta u\|_{L^{2}(\Omega)}^{2}+2 \sum_{j=1}^{N} \int_{\Gamma_{j}}\left(\gamma_{j} D_{x} u\right)\left(\gamma_{j} \frac{\partial}{\partial \tau_{j}} D_{y} u\right) d \sigma .
\end{aligned}
$$

Hence we will get (3.6) if we prove that there exists a constant $C$ such that for all $u \in T^{m}(\Omega)$ and $\varepsilon \in(0,1)$,

$$
\left|\sum_{j=1}^{N} \int_{\Gamma_{j}}\left(\gamma_{j} D_{x} u\right)\left(\gamma_{j} \frac{\partial}{\partial \tau_{j}} D_{y} u\right) d \sigma\right| \leq C\left\{\varepsilon\|u\|_{H^{2}(\Omega)}^{2}+\varepsilon^{-1}\|u\|_{H^{1}(\Omega)}^{2}\right\} .
$$

REMARK 2. The inequality (3.7) depends essentially on the boundary conditions, because its left hand side contains the traces of the second derivatives and it may be estimated by the norm of $u$ in $H^{s}$ for $s>5 / 2$. If there is no Robin boundary condition, then (3.7) holds with $C=0$ (see $[4$, Lemma 2.2.1]).

We only check (3.7) in a special case, because in the remaining cases we can proceed in the same way. Assume that $j \in \mathbf{N}, j+1 \in \mathbf{R}$ and that $\nu_{j}^{x}, \nu_{j+1}^{x} \neq 0$, where $\nu_{j}=\left(\nu_{j}^{x}, \nu_{j}^{y}\right)$. We shall estimate the quantities

$$
\int_{\Gamma_{j}}\left(\gamma_{j} D_{x} u\right)\left(\gamma_{j} \frac{\partial}{\partial \tau_{j}} D_{y} u\right) d \sigma+\int_{\Gamma_{j+1}}\left(\gamma_{j+1} D_{x} u\right)\left(\gamma_{j+1} \frac{\partial}{\partial \tau_{j+1}} D_{y} u\right) d \sigma
$$

The boundary conditions on $\Gamma_{j}$ and $\Gamma_{j+1}$ are

$$
\left\{\begin{array}{l}
\nu_{j}^{x} \gamma_{j}\left(D_{x} u\right)+\nu_{j}^{y} \gamma_{j}\left(D_{y} u\right)=0 \\
\nu_{j+1}^{x} \gamma_{j+1}\left(D_{x} u\right)+\nu_{j+1}^{y} \gamma_{j+1}\left(D_{y} u\right)=-\alpha_{j+1} u
\end{array}\right.
$$

Substituting (3.9) in (3.8) we obtain

$$
\begin{aligned}
& -\frac{\nu_{j}^{y}}{\nu_{j}^{x}} \int_{\Gamma_{j}}\left(\gamma_{j} D_{y} u\right)\left(\gamma_{j} \frac{\partial}{\partial \tau_{j}} D_{y} u\right) d \sigma-\frac{\nu_{j+1}^{y}}{\nu_{j+1}^{x}} \int_{\Gamma_{j+1}}\left(\gamma_{j+1} D_{y} u\right)\left(\gamma_{j+1} \frac{\partial}{\partial \tau_{j+1}} D_{y} u\right) d \sigma \\
& -\frac{\alpha_{j+1}}{\nu_{j+1}^{x}} \int_{\Gamma_{j+1}} \gamma_{j+1} u \cdot \gamma_{j+1}\left(\frac{\partial}{\partial \tau_{j+1}} D_{y} u\right) d \sigma .
\end{aligned}
$$

Integrating the first two expressions, and integrating the last one by parts, we get

$$
\begin{array}{rl}
\frac{1}{2}\left[\frac{\nu_{j+1}^{y}}{\nu_{j+1}^{x}}-\frac{\nu_{j}^{y}}{\nu_{j}^{x}}\right] D_{y} & u\left(S_{j+1}\right)-\frac{\alpha_{j+1}}{\nu_{j+1}^{x}} u\left(S_{j+1}\right) D_{y} u\left(S_{j+1}\right) \\
+ & \frac{\alpha_{j+1}}{\nu_{j+1}^{x}} \int_{\Gamma_{j+1}} \gamma_{j+1}\left(\frac{\partial}{\partial \tau_{j+1}} u\right) \gamma_{j+1}\left(D_{y} u\right) d \sigma+R
\end{array}
$$

where $R$ involves the vertices $S_{j}$ and $S_{j+2}$. Applying [4, Theorem 1.5.1.10] 
we can estimate the integral in (3.10) by the right hand side of (3.7). If $\nu_{j+1}^{y} / \nu_{j+1}^{x}-\nu_{j}^{y} / \nu_{j}^{x} \neq 0$, then $\operatorname{det}\left(\nu_{j}, \nu_{j+1}\right) \neq 0$, hence from (3.9) we get $D_{y} u\left(S_{j+1}\right)=c_{0} u\left(S_{j+1}\right)$, where the constant $c_{0}$ depends only on the boundary conditions. Therefore, the first two terms in (3.10) are equal to $c_{1} u^{2}\left(S_{j+1}\right)$ for a constant $c_{1}$. Now, using the Sobolev embedding theorem and an interpolation inequality, we obtain the estimate in the form (3.7).

If $\nu_{j+1}^{y} / \nu_{j+1}^{x}-\nu_{j}^{y} / \nu_{j}^{x}=0$, then the left hand sides of (3.9) are equal at $S_{j+1}$ ([4, Theorem 1.6.1.5]), hence so are the right hand sides, and thus $u\left(S_{j+1}\right)=0$. This means that the first two terms in (3.10) vanish in this case. Clearly, the terms involving $S_{j}$ and $S_{j+2}$ will be cancelled by appropriate terms coming from the integrals on $\Gamma_{j-1}$ and $\Gamma_{j+2}$.

REMARK 3. We have chosen the special case $j \in \mathbf{N}, j+1 \in \mathbf{R}$ and $\nu_{j}^{x}, \nu_{j+1}^{x} \neq 0$ in order to show the idea of the proof of (3.7). In all other cases we proceed in the same way. The details are omitted.

3.2. Proof of Lemma 2. First we reduce the density of $T^{m}(\Omega)$ in $T^{2}(\Omega)$ to the appropriate density on $\partial \Omega$. Set

$$
\gamma:=\left(\gamma_{j}, \gamma_{j} \frac{\partial}{\partial \nu_{j}}\right)_{j=1}^{N}, \quad Z^{m}:=\gamma\left(H^{m}(\Omega) \cap T^{2}(\Omega)\right) .
$$

Thus, $Z^{2}(\Omega)$ is a subspace of $\prod_{j=1}^{N} H^{3 / 2}\left(\Gamma_{j}\right) \times H^{1 / 2}\left(\Gamma_{j}\right)\left(Z^{2}(\Omega)\right.$ being equipped with its natural image topology). Hence the operator $(\Pi, \gamma)$, where $\Pi$ is the projection of $T^{2}(\Omega)$ onto $H_{0}^{2}(\Omega)$, is an isomorphism from $T^{2}(\Omega)$ onto $H_{0}^{2}(\Omega) \times Z^{2}(\Omega)$, i.e.

$$
T^{2}(\Omega) \cong H_{0}^{2}(\Omega) \times Z^{2}(\Omega) .
$$

Thus, the density of $T^{m}(\Omega)$ for $m \geq 3$ in $T^{2}(\Omega)$ reduces to the density of $Z^{m}$ for $m \geq 3$ in $Z^{2}(\Omega)$. Without loss of generality we may assume that $m \geq 4$.

In order to prove the desired density, first we have to describe the spaces of traces $Z^{2}(\Omega)$ and $Z^{m}$ for $m \geq 4$. We will need the following notations. If $f \in H^{1 / 2}\left(\Gamma_{j-1}\right), g \in H^{1 / 2}\left(\Gamma_{j}\right)$, then we write

$$
\begin{aligned}
f \equiv_{S_{j}} g & \Leftrightarrow f\left(x_{j}(-\sigma)\right)-g\left(x_{j}(\sigma)\right) \in \widetilde{H}^{1 / 2}\left(\mathbb{R}_{+}\right), \\
(f, g)_{\mathfrak{R}_{\delta}} & :=\int_{0}^{\delta} \frac{\left|f\left(x_{j}(-\sigma)\right)-g\left(x_{j}(\sigma)\right)\right|^{2}}{\sigma} d \sigma .
\end{aligned}
$$

[5, Lemma 1.6.3] gives the following description of $Z^{2}(\Omega):\left\langle f_{j}, g_{j}\right\rangle_{j=1}^{N} \in$ $Z^{2}(\Omega)$ if and only if $\left\langle f_{j}, g_{j}\right\rangle_{j=1}^{N} \in \prod_{j=1}^{N} H^{3 / 2}\left(\Gamma_{j}\right) \times H^{1 / 2}\left(\Gamma_{j}\right)$ and for $j \in$ 
$\{1, \ldots, N\}$ we have

$$
\begin{cases}f_{j}=0 & \text { if } j \in \mathbf{D}, \\ g_{j}=0 & \text { if } j \in \mathbf{N}, \\ g_{j}+\alpha_{j} f_{j}=0 & \text { if } j \in \mathbf{R},\end{cases}
$$

(a) $f_{j-1}\left(S_{j}\right)=f_{j}\left(S_{j}\right)$,

(b $) \frac{\partial}{\partial \tau_{j-1}} f_{j-1} \equiv S_{j}-\cos \omega_{j} \frac{\partial}{\partial \tau_{j}} f_{j}+\sin \omega_{j} g_{j}$,

$\left(\mathrm{b}_{2}\right) g_{j-1} \equiv S_{j}-\cos \omega_{j} g_{j}-\sin \omega_{j} \frac{\partial}{\partial \tau_{j}} f_{j}$.

The space $Z^{m}$ for $m \geq 4$ is described by conditions (3.12) and in addition each $\left\langle f_{j}, g_{j}\right\rangle_{j=1}^{N} \in Z^{m}$ satisfies $\left\langle f_{j}, g_{j}\right\rangle_{j=1}^{N} \in \prod_{j=1}^{N} H^{m-1 / 2}\left(\Gamma_{j}\right) \times H^{m-3 / 2}\left(\Gamma_{j}\right)$ and for $j=1, \ldots, N$ we have

$$
\begin{aligned}
\cos \omega_{j} \frac{\partial^{2}}{\partial \tau_{j-1}^{2}} f_{j-1}\left(S_{j}\right)+\sin \omega_{j} & \frac{\partial}{\partial \tau_{j-1}} g_{j-1}\left(S_{j}\right) \\
& =\cos \omega_{j} \frac{\partial^{2}}{\partial \tau_{j}^{2}} f_{j}\left(S_{j}\right)-\sin \omega_{j} \frac{\partial}{\partial \tau_{j}} g_{j}\left(S_{j}\right) .
\end{aligned}
$$

It can be easily verified that the topology on $Z^{2}(\Omega)$ is induced by the norm

$$
\begin{aligned}
\left\|\left\langle f_{j}, g_{j}\right\rangle_{j=1}^{N}\right\|_{\gamma}:= & \left\{\sum_{j=1}^{N}\left\|\left\langle f_{j}, g_{j}\right\rangle_{j=1}^{N}\right\|_{H^{3 / 2}\left(\Gamma_{j}\right) \times H^{1 / 2}\left(\Gamma_{j}\right)}^{2}\right. \\
& +\left(\frac{\partial}{\partial \tau_{j-1}} f_{j-1},-\cos \omega_{j} \frac{\partial}{\partial \tau_{j}} f_{j}+\sin \omega_{j} g_{j}\right)_{\mathfrak{R}_{\delta}} \\
& \left.+\left(g_{j-1},-\cos \omega_{j} g_{j}-\sin \omega_{j} \frac{\partial}{\partial \tau_{j}} f_{j}\right)_{\mathfrak{R}_{\delta}}\right\}^{1 / 2} .
\end{aligned}
$$

Here $0<\delta<\min _{j}\left|\Gamma_{j}\right|$ and $(\cdot, \cdot)_{\mathfrak{R}_{\delta}}$ was defined in (3.11). Thus we have to prove the density of $Z^{m}$ in $Z^{2}(\Omega)$ with respect to the norm $\|\cdot\|_{\gamma}$. Using a partition of unity the problem can be reduced to the appropriate density in some neighbourhoods of the vertices $S_{j}, j=1, \ldots, N$. We will show it only in the special case: $j-1, j \in \mathbf{R}$. In the remaining cases we proceed in the same way. First we formulate an obvious property of the Sobolev space $H^{3 / 2}\left(\mathbb{R}_{+}\right)$:

Proposition 2. If $f \in H^{3 / 2}\left(\mathbb{R}_{+}\right)$and $f^{\prime} \in \widetilde{H}^{1 / 2}\left(\mathbb{R}_{+}\right)$, then for every $a \in \mathbb{R}$ there exists a sequence $\left\{f_{n}\right\} \subseteq \mathcal{D}\left(\overline{\mathbb{R}}_{+}\right)$such that $f_{n}-f \rightarrow 0$ in $\widetilde{H}^{3 / 2}\left(\mathbb{R}_{+}\right)$as $n \rightarrow \infty$ and for all $n \in \mathbb{N}$ we have $f_{n}(0)=f(0), f_{n}^{\prime}(0)=0$ and $f_{n}^{\prime \prime}(0)=a$. 
We introduce the following notations: $\omega:=\omega_{j}, p_{0}(\sigma):=f_{j}\left(x_{j}(-\sigma)\right)$, $q_{0}(\sigma):=f_{j+1}\left(x_{j}(-\sigma)\right), p_{1}(\sigma):=g_{j}\left(x_{j}(-\sigma)\right), q_{1}(\sigma):=g_{j+1}\left(x_{j}(-\sigma)\right)$. Then the problem of the density in a neighbourhood of $S_{j}$ for $j-1, j \in \mathbf{R}$ has the following form: we need to approximate functions $\left(p_{k}, q_{k}\right)_{k=0,1} \in$ $\prod_{k=0}^{1} H^{3 / 2-k}\left(\mathbb{R}_{+}\right) \times H^{3 / 2-k}\left(\mathbb{R}_{+}\right)$satisfying

$$
\begin{aligned}
& \text { (a) } p_{0}(0)=q_{0}(0) \text {, } \\
& \text { ( } \left.\mathrm{b}_{1}\right) \quad p_{0}^{\prime}-\cos \omega q_{0}^{\prime}-\alpha_{2} \sin \omega q_{0} \in \widetilde{H}^{1 / 2}\left(\mathbb{R}_{+}\right) \text {, } \\
& \left(\mathrm{b}_{2}\right)-\alpha_{1} p_{0}-\alpha_{2} \cos \omega q_{0}+\sin \omega q_{0}^{\prime} \in \widetilde{H}^{1 / 2}\left(\mathbb{R}_{+}\right) \text {, } \\
& \text { (d) } p_{1}+\alpha_{1} p_{0}=0 \text {, } \\
& \text { (d2) } q_{1}+\alpha_{2} q_{0}=0,
\end{aligned}
$$

by functions $\left(p_{k}^{n}, q_{k}^{n}\right)_{k=0,1} \in \prod_{k=0}^{1} H^{3 / 2-k+m}\left(\mathbb{R}_{+}\right) \times H^{3 / 2-k+m}\left(\mathbb{R}_{+}\right)$satisfying (3.15) and in addition

$$
-\cos \omega p_{0}^{n \prime \prime}(0)+\sin \omega p_{1}^{n \prime}(0)=-\cos \omega q_{0}^{n \prime \prime}(0)+\sin \omega q_{1}^{n \prime}(0) .
$$

The approximation should be with respect to the norm of $\prod_{j=1}^{N} H^{3 / 2}\left(\Gamma_{j}\right) \times$ $H^{1 / 2}\left(\Gamma_{j}\right)$ and such that

$$
\begin{aligned}
& \left(p_{0}^{\prime}-\cos \omega q_{0}^{\prime}+\sin \omega q_{1}, p_{0}^{n \prime}-\cos \omega q_{0}^{n \prime}+\sin \omega q_{1}^{n}\right)_{\mathfrak{R}} \underset{n \rightarrow \infty}{\longrightarrow} 0, \\
& \left(p_{1}+\cos \omega q_{1}+\sin \omega q_{0}{ }^{\prime}, p_{1}^{n}+\cos \omega q_{1}^{n}+\sin \omega q_{0}^{n \prime}\right)_{\mathfrak{R}} \underset{n \rightarrow \infty}{\longrightarrow} 0 .
\end{aligned}
$$

Here $(f, g)_{\Re}=\int_{\mathbb{R}_{+}} \frac{|f(t)-g(t)|^{2}}{t} d t$. From (a) and $\left(\mathrm{b}_{2}\right)$ of (3.15) we have

$$
\left(\alpha_{1}+\alpha_{2} \cos \omega\right) q_{0}-\sin \omega q_{0}^{\prime} \in \widetilde{H}^{1 / 2}\left(\mathbb{R}_{+}\right) .
$$

To get (3.16), we assume that

$$
\frac{\cos \omega}{\sin \omega}\left(c_{1}-c_{2}\right)=\left(\left(\alpha_{1}^{2}+\alpha_{2}^{2}\right) \cos \omega+2 \alpha_{1} \alpha_{2}\right) q_{0}(0)
$$

for some $c_{1}, c_{2} \in \mathbb{R}$. Let $h(t):=\left(\alpha_{1}+\alpha_{2} \cos \omega\right) q_{0}(0) t \psi(t)-\sin \omega q_{0}(t)$, where $\psi$ is a smooth function such that $\psi \equiv 1$ on $B(0, \varepsilon)$ and $\psi \equiv 0$ outside $B(0,2 \varepsilon)$ for some $\varepsilon>0$. Then from (3.17) we have $h^{\prime} \in \widetilde{H}^{1 / 2}\left(\mathbb{R}_{+}\right)$, and hence from Proposition 2 we get a sequence $\left\{h_{n}\right\}_{n \in \mathbb{N}} \subseteq \mathcal{D}\left(\overline{\mathbb{R}}_{+}\right)$such that

$$
\begin{aligned}
& h_{n}-h \underset{n \rightarrow \infty}{\longrightarrow} 0 \text { in } \widetilde{H}^{3 / 2}\left(\mathbb{R}_{+}\right), \\
& h_{n}(0)=-\sin \omega q_{0}(0), \quad h_{n}^{\prime}(0)=0, \quad h_{n}^{\prime \prime}(0)=c_{1} .
\end{aligned}
$$

From $(a),\left(b_{1}\right)$ and $\left(b_{2}\right)$ we obtain

$$
\left(\alpha_{1} \cos \omega+\alpha_{2}\right) p_{0}-\sin \omega p_{0}^{\prime} \in \widetilde{H}^{1 / 2}\left(\mathbb{R}_{+}\right) .
$$

Set $w(t):=\left(\alpha_{1} \cos \omega+\alpha_{2}\right) p_{0}(0) t \psi(t)-\sin \omega p_{0}(t)$. Then from (3.20) we 
have $w^{\prime} \in \widetilde{H}^{1 / 2}\left(\mathbb{R}_{+}\right)$and applying again Proposition 2 we find a sequence $\left\{w_{n}\right\}_{n \in \mathbb{N}} \subseteq \mathcal{D}\left(\overline{\mathbb{R}}_{+}\right)$such that

$$
\begin{aligned}
& w_{n} \underset{n \rightarrow \infty}{\longrightarrow} w \text { in } \widetilde{H}^{3 / 2}\left(\mathbb{R}_{+}\right), \\
& w_{n}(0)=-\sin \omega p_{0}(0), \quad w_{n}^{\prime}(0)=0, \quad w_{n}^{\prime \prime}(0)=c_{2} .
\end{aligned}
$$

We have assumed that $\omega \neq \pi$, hence we can define

$$
\begin{array}{ll}
p_{0}^{n}:=\frac{1}{\sin \omega}\left(\left(\alpha_{1} \cos \omega+\alpha_{2}\right) q_{0}(0) t \psi-w_{n}\right), & p_{1}^{n}:=-\alpha_{1} p_{0}^{n}, \\
q_{0}^{n}:=\frac{1}{\sin \omega}\left(\left(\alpha_{2} \cos \omega+\alpha_{1}\right) q_{0}(0) t \psi-h_{n}\right), & q_{1}^{n}:=-\alpha_{2} q_{0}^{n} .
\end{array}
$$

It is clear $\left(p_{k}^{n}, q_{k}^{n}\right)_{k=0,1} \in \prod_{k=0}^{1} H^{3 / 2-k+m}\left(\mathbb{R}_{+}\right) \times H^{3 / 2-k+m}\left(\mathbb{R}_{+}\right)$and from the definition of $p_{0}^{n}, q_{0}^{n}, h_{n}$ and $w_{n}$ we get

$$
\begin{aligned}
& \left\|p_{0}-p_{0}^{n}\right\|_{\widetilde{H}^{3 / 2}\left(\mathbb{R}_{+}\right)}=\left|\frac{1}{\sin \omega}\right|\left\|w-w_{n}\right\|_{\widetilde{H}^{3 / 2}\left(\mathbb{R}_{+}\right)} \underset{n \rightarrow \infty}{\longrightarrow} 0 \\
& \left\|q_{0}-q_{0}^{n}\right\|_{\widetilde{H}^{3 / 2}\left(\mathbb{R}_{+}\right)}=\left|\frac{1}{\sin \omega}\right|\left\|h-h_{n}\right\|_{\widetilde{H}^{3 / 2}\left(\mathbb{R}_{+}\right)} \underset{n \rightarrow \infty}{\longrightarrow} 0 .
\end{aligned}
$$

Thus, we have the desired convergence. It can be easily verified that conditions $(\mathrm{a}),\left(\mathrm{b}_{1}\right),\left(\mathrm{b}_{2}\right)$ of $(3.15)$ are satisfied and the equality (3.16) holds, because $c_{1}$ and $c_{2}$ were defined by (3.18). Clearly, the boundary conditions are also satisfied, hence the sequence $\left(p_{k}^{n}, q_{k}^{n}\right)_{k=0,1}$ is the desired approximation of $\left(p_{k}, q_{k}\right)_{k=0,1}$.

In the remaining cases of the boundary conditions we proceed similarly. The details are omitted.

REMARK 4. For other boundary conditions we also apply the following property of Sobolev spaces:

Proposition 3. If $f \in H^{3 / 2}\left(\mathbb{R}_{+}\right)$, then there exists a sequence $\left\{f_{n}\right\} \subseteq$ $\mathcal{D}\left(\overline{\mathbb{R}}_{+}\right)$such that $f_{n} \rightarrow f$ in $H^{3 / 2}\left(\mathbb{R}_{+}\right)$as $n \rightarrow \infty$ and for all $n \in \mathbb{N}$ we have $f_{n}(0)=f(0), f_{n}^{\prime}(0)=a_{1}, f_{n}^{\prime \prime}(0)=a_{2}$, where $a_{1}, a_{2} \in \mathbb{R}$ are arbitrary given numbers. Furthermore, the imbedding $H_{0}^{3 / 2}\left(\mathbb{R}_{+}\right) \hookrightarrow \widetilde{H}^{1 / 2}\left(\mathbb{R}_{+}\right)$is continuous.

Thus, we have proved inequality (3.4), therefore the proof of Theorem 3 is complete.

4. Description of the annihilator $\mathcal{N}$. The goal of this section is to describe the annihilator of $\Delta T^{2}(\Omega)$, denoted by $\mathcal{N}$ and defined by

$$
v \in \mathcal{N} \Leftrightarrow v \in L^{2}(\Omega) \text { and } \int_{\Omega} v \Delta u d x d y=0 \text { for all } u \in T^{2}(\Omega) .
$$


For each $j=1, \ldots, N$ define

$$
\widetilde{\psi}_{j}:= \begin{cases}\exp \left(A_{j} x_{j}+B_{j} y_{j}\right) & \text { for } j-1, j \in \mathbf{N} \cup \mathbf{R}, \\ y_{j} \exp \left(A_{j} x_{j}\right) & \text { for } j-1 \in \mathbf{D}, j \in \mathbf{N} \cup \mathbf{R}, \omega_{j}=\pi / 2,3 \pi / 2, \\ x_{j} \exp \left(B_{j} y_{j}\right) & \text { for } j-1 \in \mathbf{N} \cup \mathbf{R}, j \in \mathbf{D}, \omega_{j}=\pi / 2,3 \pi / 2, \\ 0 & \text { in the other cases }\end{cases}
$$

where $A_{j}$ and $B_{j}$ are so chosen that $\widetilde{\psi}_{j}$ satisfies the boundary conditions on $\Gamma_{j-1}$ and $\Gamma_{j}$. Furthermore, let $\eta_{j}=\eta\left(r_{j}\right)$ be smooth functions such that $\eta_{j} \equiv 1$ on $B\left(S_{j}, \varepsilon\right)$ and $\eta_{j} \equiv 0$ outside $B\left(S_{j}, 2 \varepsilon\right)$, for some positive $\varepsilon<\min _{j}\left|\Gamma_{j}\right| / 4$. Then we define

$$
\psi_{j}:=\eta_{j} \widetilde{\psi}_{j}
$$

It is clear that the functions $\psi_{j}$ for $j=1, \ldots, N$ are smooth on $\bar{\Omega}$ and satisfy the boundary conditions of $(2.1)$. Hence $\psi_{j} \in T^{2}(\Omega)$ for $j=1, \ldots, N$. Moreover, the functions $\psi_{j}$ have the following property: if $u \in T^{3}(\Omega)$, then the function

$$
w_{u}:=u-\sum_{j \in J_{1}} u\left(S_{j}\right) \psi_{j}-\sum_{j \in J_{2}} \frac{\partial u}{\partial y_{j}}\left(S_{j}\right) \psi_{j}-\sum_{j \in J_{3}} \frac{\partial u}{\partial x_{j}}\left(S_{j}\right) \psi_{j}
$$

satisfies

$$
w_{u}\left(S_{j}\right)=0, \quad \nabla w_{u}\left(S_{j}\right)=0 \quad \text { for } j=1, \ldots, N,
$$

where

$$
\begin{aligned}
& J_{1}:=\{j ; j-1, j \in \mathbf{N} \cup \mathbf{R}\}, \\
& J_{2}:=\left\{j ; j-1 \in \mathbf{D}, j \in \mathbf{N} \cup \mathbf{R}, \omega_{j}=\pi / 2,3 \pi / 2\right\}, \\
& J_{3}:=\left\{j ; j-1 \in \mathbf{N} \cup \mathbf{R}, j \in \mathbf{D}, \omega_{j}=\pi / 2,3 \pi / 2\right\} .
\end{aligned}
$$

By straightforward calculation we also have

$$
\frac{\partial^{i}}{\partial \theta_{j}^{i}} \psi_{j}=r_{j} \psi_{j} W_{i, j}\left(\cos \omega_{j}, \sin \omega_{j}, r_{j}\right) \quad \text { on } \Gamma_{j} \text { for } j \in \mathbf{N} \cup \mathbf{R},
$$

where $W_{i, j}$ are some polynomials in $\cos \omega_{j}, \sin \omega_{j}, r_{j}$, and $i=1,2$. Using the above notations we now characterize the annihilator $\mathcal{N}$.

THEOREM 4. The elements of the annihilator $\mathcal{N}$ are the very weak solutions of the homogeneous problem (2.1) which are orthogonal to the Laplacian of $\psi_{j}$, i.e.

$$
v \in \mathcal{N} \Leftrightarrow v \in \mathcal{M} \text { and } \int_{\Omega} v \Delta \psi_{j} d x d y=0 \text { for } j=1, \ldots, N .
$$

REMARK 5. It is worth stressing that the above orthogonality conditions are essential. As will be shown in Theorems 2 and 3 of [9], the annihilator $\mathcal{N}$ is a proper subspace of $\mathcal{M}$ if $j-1, j \in \mathbf{N} \cup \mathbf{R}$ for some $j$. 
Proof of Theorem 4. Assume that $v \in \mathcal{N}$; then by (4.1) we have $\int_{\Omega} v \Delta \psi_{j} d x d y=0$ and $\Delta v=0$ in the sense of distibutions, because $\psi_{j} \in$ $T^{2}(\Omega)$ and $\mathcal{D}(\Omega) \subseteq T^{2}(\Omega)$. Now we check that $v$ satisfies the boundary conditions of (2.1). From the trace theorem 1.4.6 of [5] we know that for any $\varphi_{j}, \phi_{j}, \xi_{j} \in \mathcal{D}\left(\Gamma_{j}\right)$ there exists a function $u \in H^{2}(\Omega)$ such that

$$
\begin{array}{llll}
\gamma_{j} u=0, & \gamma_{j} \frac{\partial}{\partial \nu_{j}} u=\varphi_{j} & \text { for } j \in \mathbf{D}, \\
\gamma_{j} u=\phi_{j}, & \gamma_{j} \frac{\partial}{\partial \nu_{j}} u=0 & \text { for } j \in \mathbf{N}, \\
\gamma_{j} u=-\xi_{j}, & \gamma_{j} \frac{\partial}{\partial \nu_{j}} u=\alpha_{j} \xi_{j} & \text { for } j \in \mathbf{R} .
\end{array}
$$

Hence $u \in T^{2}(\Omega)$ and we can use the Green formula ([4, Theorem 1.5.3]) for $u$ and $v$, which by the assumption reduces to

$$
-\sum_{j \in \mathbf{D}}\left\langle\gamma_{j} v, \varphi_{j}\right\rangle+\sum_{j \in \mathbf{N}}\left\langle\gamma_{j} \frac{\partial}{\partial \nu_{j}} v, \phi_{j}\right\rangle+\sum_{j \in \mathbf{R}}\left\{\left\langle\gamma_{j} \frac{\partial}{\partial \nu_{j}} v,-\xi_{j}\right\rangle-\left\langle\gamma_{j} v, \alpha_{j} \xi_{j}\right\rangle\right\}=0 .
$$

The functions $\varphi_{j}, \phi_{j}, \xi_{j} \in \mathcal{D}\left(\Gamma_{j}\right)$ can be chosen independently. Thus from the density of $\mathcal{D}\left(\Gamma_{j}\right)$ in both $\widetilde{H}^{1 / 2}\left(\mathbb{R}_{+}\right)$and $\widetilde{H}^{3 / 2}\left(\mathbb{R}_{+}\right)$(see $[4$, Theorem 1.4.2.1]) we conclude that $v$ satisfies the boundary conditions in (2.1), therefore $v \in \mathcal{M}$.

Conversely, assume that $v \in \mathcal{M}$ and $\int_{\Omega} v \Delta \psi_{j} d x d y=0$ for $j=1, \ldots, N$. We will show that

$$
\int_{\Omega} v \Delta u d x d y=0 \quad \text { for } u \in T^{3}(\Omega) .
$$

Hence applying the density of $T^{3}(\Omega)$ in $T^{2}(\Omega)$ (Lemma 2) we obtain $v \in \mathcal{N}$. To prove (4.7) take an arbitrary $u \in T^{3}(\Omega)$ and let $w_{u}$ be as in (4.4). From the assumptions we have

$$
\int_{\Omega} v \Delta u d x d y=\int_{\Omega} v \Delta w_{u} d x d y
$$

Now (4.5) allows us to apply the Green formula ([5, Theorem 1.5.3]) for $v$ and $w_{u}$, which reduces to the equality $\int_{\Omega} v \Delta w_{u} d x d y=0$, because $\Delta v=0$ and both $v$ and $w_{u}$ satisfy the boundary conditions (2.1). Hence, from (4.8) we obtain (4.7), thus $v \in \mathcal{N}$ and the proof is finished.

REMARK 6 . From the proof of Theorem 4 we see that the orthogonality conditions were necessary, because we would be unable to apply the Green formula ([5, Theorem 1.5.3]) for $v \in D\left(\Delta, L^{2}(\Omega)\right)$ and $u \in H^{2}(\Omega)$ if $u$ does not vanish in some neighbourhood of the vertices $S_{j}, j=1, \ldots, N$. For some boundary conditions the Green formula can be generalized (see [1]). Then 
the traces can be defined in such a way that the annihilator $\mathcal{N}$ is exactly the space of solutions of the homogeneous formal adjoint problem.

In the second part [9] we will examine the space of very weak solutions of the homogeneous problem. This will allow us to finish the proof of Theorems 1 and 2. Furthermore, we will formulate an analogous result for nonhomogeneous boundary conditions.

\section{References}

[1] J. Banasiak, On $L^{2}$-solvability of mixed boundary value problems for elliptic equations in plane non-smooth domains, J. Differential Equations 97 (1992), 99-111.

[2] M. Dauge, Elliptic Boundary Value Problems on Corner Domains. Smoothness and Asymptotics of Solutions, Lecture Notes in Math. 1341, Springer, Berlin, 1988.

[3] H. Ding, Very weak solutions of boundary value problems for the Laplace operator and the Lamé system on polyhedral domains in $\mathbb{R}^{3}$, Proc. Roy. Soc. Edinburgh Sect. A 124 (1994), 645-672.

[4] P. Grisvard, Elliptic Problems in Nonsmooth Domains, Pitman, London, 1985.

[5] - Singularities in Boundary Value Problems, Masson, Paris, 1992.

[6] V. A. Kondrat'ev, Boundary value problems for elliptic equations in domains with conical and angular points, Trans. Moscow Math. Soc. 16 (1967), 227-313.

[7] V. A. Kozlov, V. G. Maz'ya and J. Rossmann, Elliptic Boundary Value Problems in Domains with Point Singularities, Math. Surveys Monogr. 52, Amer. Math. Soc., Providence, RI, 1997.

[8] A. Kubica, Poisson equation on a polygonal domain with mixed boundary conditions (in Polish), Master Thesis, Warszawa 2003.

[9] - The regularity of weak and very weak solutions of the Poisson equation on polygonal domains with mixed boundary conditions (part II), Appl. Math. (Warsaw), to appear.

[10] Z. Mghazli, Regularity of an elliptic problem with mixed Dirichlet-Robin boundary conditions in a polygonal domain, Calcolo 29 (1992), 241-267 (1993).

[11] S. A. Nazarov and B. A. Plamenevsky, Elliptic Problems in Domains with Piecewise Smooth Boundaries, de Gruyter Exp. Math. 13, de Gruyter, Berlin, 1994.

[12] J. Nečas, Les Méthodes Directes en Théorie des Équations Elliptiques, Masson, Paris, 1967.

[13] S. Rempel and B.-W. Schulze, Asymptotics for Elliptic Mixed Boundary Problems. Pseudo-Differential and Mellin Operators in Spaces with Conormal Singularity, Math. Res. 50, Akademie-Verlag, Berlin, 1989.

Institute of Mathematics

Polish Academy of Sciences

Śniadeckich 8

00-956 Warszawa, Poland

E-mail: Adam.Kubica@zodiac.mimuw.edu.pl

Received on 7.6.2004;

revised version on 30.8 .2004 